\title{
Innovation and Trends with In-Memory Technology
}

\section{Matthias Steinbrecher}

Innovation Center Potsdam, TIP HPI Strategic Projects SAP AG, Potsdam, Germany http://www.sap.com

High performance in-memory computing will change how enterprises work. Currently, enterprise data is split into two databases for performance reasons. Usually, disk-based row-oriented database systems are used for operational data and column-oriented databases are used for analytics. Since hardware architectures have evolved dramatically during the past decade, this scenario has now changed. Multi-core architectures and the availability of large amounts of main memory at low costs are about to set new breakthroughs in the software industry. Traditional disks are one of the last remaining mechanical devices in a world of silicon and are about to become what tape drives are today: a device only necessary for backup. With in-memory computing and hybrid databases using both row and column-oriented storage where appropriate, transactional and analytical processing can be unified, allowing data analysis algorithms to run inside the database.

Relevant Web sites

1. http://www.sap.com/ 